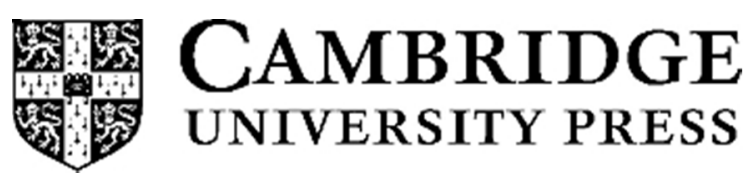

\title{
SIW Antennas as Hybrid Energy Harvesting and Power Management Platforms for the Internet of Things
}

\begin{tabular}{|r|l|}
\hline Journal: & International Journal of Microwave and Wireless Technologies \\
\hline Manuscript ID & MRF-RP-15-404.R1 \\
\hline Manuscript Type: & Research Paper \\
\hline Date Submitted by the Author: & 11 -Feb-2016 \\
\hline Complete List of Authors: & $\begin{array}{l}\text { Caytan, Olivier; Ghent University/iMinds, Information Technology } \\
\text { Lemey, Sam; Ghent University/iMinds, Information Technology } \\
\text { Agneessens, Sam; Ghent University/iMinds, Information Technology } \\
\text { Rogier, Hendrik; Ghent University/iMinds, Information Technology }\end{array}$ \\
\hline Topics: & $\begin{array}{l}\text { Antenna Design, Modelling and Measurements, Antennas and Propagation } \\
\text { for Wireless Systems }\end{array}$ \\
\hline Additional Topic (optional): & \multicolumn{2}{|c|}{} \\
\hline \multicolumn{2}{|c|}{} \\
\hline
\end{tabular}

\section{SCHOLARONE"}

Manuscripts 


\title{
SIW Antennas as Hybrid Energy Harvesting and Power Management Platforms for the Internet of Things
}

\author{
Olivier Caytan ${ }^{1}$, Sam Lemey ${ }^{1}$, Sam Agneessens ${ }^{1}$, Hendrik Rogier ${ }^{1}$ \\ ${ }^{1}$ Electromagnetics Group, Department of Information Technology, Ghent \\ University/iMinds, Sint-Pietersnieuwstraat 41, 9000 Ghent, Belgium
}

\begin{abstract}
A novel antenna-harvester co-design paradigm is presented for wireless nodes operating in an Internet of Things context. The strategy leads to compact and highly-integrated units, which are able to set up a reliable and energy-efficient wireless communication link, and to simultaneously harvest energy from up to three different sources, including thermal body energy, solar and artificial light. The core of the unit consists of a substrate-integrated-waveguide (SIW) antenna. Its surface serves as a platform for the flexible energy harvesting hardware, which also comprises the power management system. To demonstrate the approach, two different SIW cavity-backed slot antennas and a novel compact dual linearly polarized SIW antenna are presented. These topologies facilitate the integration of additional hardware without degrading performance. In the meantime, they enable comfortable integration into garments or unobtrusive embedding into floors or walls. Measurements on prototypes validate the integration procedure by verifying that the integrated hardware has a negligible influence on the performance of all discussed SIW antennas. Finally, measurements in four well-chosen indoor scenarios demonstrate that a hybrid energy-harvesting approach is necessary to obtain a more continuous flow and a higher amount of scavenged energy, leading to a higher system autonomy and/or reduced battery size.
\end{abstract}

Keywords:

Corresponding author: Olivier Caytan; email: olivier.caytan@intec.ugent.be; phone: +32 92643323

\section{INTRODUCTION}

In the near future, it is expected that everyday objects, tools and garments will be integrated in the Internet. This vision is coined the "Internet of Things" (IoT). The utility of everyday objects will be augmented considerably by integrating electronic components that enable computing, communication and interaction with the environment [1]. In particular, smart fabric interactive textile (SFIT) systems, in which the functionality of garments is increased without adding heavy or cumbersome equipment, are starting to find their way into a growing amount of real-life applications [2]. For instance, in critical professional applications, SFIT systems enable monitoring, localization and coordination of first responders during emergencies. Employing SFIT systems in health-care applications facilitates wireless monitoring of 
vital signs of patients in hospitals or in home-care scenarios. A major issue of current-generation SFIT and other IoT related systems concerns the limited system autonomy [1] [3]. Lots of research efforts were already devoted to increase efficiency of the embedded electronic circuitry in order to increase system autonomy without using large and obtrusive batteries. As [4] indicates that the wireless communication unit is the major power consumer, a large increase in system autonomy can be obtained by carefully designing the antenna for large radiation efficiency and high gain. Also, multiple highly-efficient antennas may be deployed onto the user's body to further increase autonomy [5]. Furthermore, energy can be harvested from ambient sources at the place where the system is deployed [6] [7], or from the user's activities [8] [9]. Preferably, energy is scavenged from multiple diverse energy sources [6] [10] to be able to harvest more energy in a more continuous way. A power management system is required in all these systems to convert the small and highly fluctuating levels of harvested energy into a form useful for powering the system. In particular, SFIT systems are prime candidates to be partly, or even solely, powered by energy harvesting techniques [10], as many physiological signals that are monitored by sensors in SFIT systems vary slowly. Accordingly, they allow burst data measurement and transmission, resulting in low duty cycle operation, yielding a low average power consumption.

Antenna topologies based on substrate-integrated-waveguide (SIW) technology [11] [12] show high potential for integration into everyday objects and garments. Their planar structure and low profile allow unobtrusive embedding in any surface, by reusing the dielectric material of the object itself, which is cost and area effective. It was recently proven that stable and high-performance SIW antennas can indeed be integrated into off-the-shelf textile fabrics and foams [13] [14] or into common floor and wall materials, such as cork [15]. More in particular, SIW cavity-backed antenna topologies [12] typically provide a high radiation efficiency and a good front-to-back ratio (FTBR), in line with energy autonomy systems. Furthermore, they exhibit a high isolation from their environment owing to the antenna cavity containing the fields, which facilitates the integration of additional hardware.

In this paper, we introduce a procedure for the integration of multiple energy harvesters and a corresponding power management module on SIW based antenna topologies, without affecting antenna performance, wearability or unobtrusive embedding. Section II outlines this procedure. First, several suitable SIW antenna topologies are discussed, intended for garment integration or for embedding in floor or wall materials. Next, we describe the integration of a flexible hybrid energy harvesting system onto these antenna topologies without disturbing their performance, enabling energy scavenging from up to three different energy sources. The performance of the system is validated in section III through measurements on prototypes. First, the integration procedure is validated by verifying that the integrated hardware has a negligible influence on the antenna performance. Second, the measurements performed in [10] are extended with additional measurements in four well-considered realistic indoor environments, emphasizing the advantages and importance of hybrid energy harvesting. 


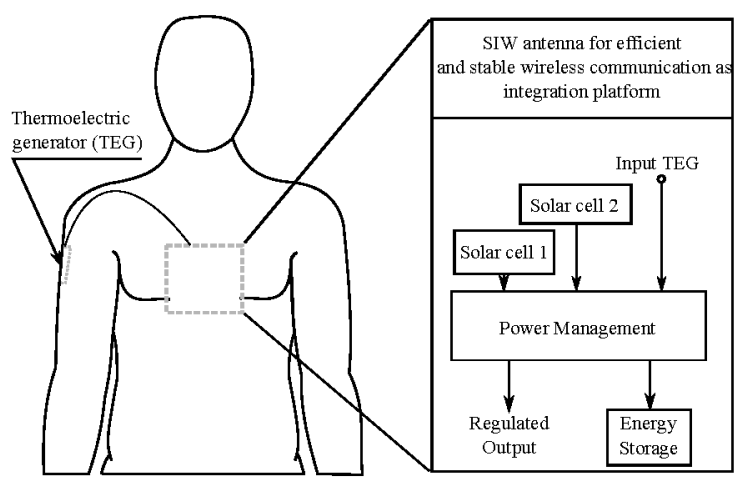

Figure 1: Block schematic representation of system concept and application.

\section{ANTENNA/HARVESTER CO-DESIGN}

We now describe the design strategy to come to a compact and highly-integrated wireless node that is able to set up a robust, stable and energy-efficient wireless communication link, and to simultaneously harvest energy from up to three different sources, in order to provide a regulated output for adequately powering the communication system. The architecture of the system is schematically outlined in Fig. 1. The core of the unit is the SIW antenna. The hardware integration strategy consists in reusing the SIW antenna's surface as a platform for the energy harvesting and power management hardware. More specifically, a flexible central power management system (CPMS), including a micro-energy cell (MEC), is deployed on the SIW antenna's backside. The CPMS enables energy harvesting from both indoor artificial light and outdoor solar light by relying on two different flexible solar cells integrated on top of the antenna. Furthermore, thermal body energy harvesting is implemented by including a thermoelectric generator (TEG), placed with its hot side on the human skin, and extending the CPMS with an ultra-low voltage step-up converter. In an IoT scenario where the antenna is integrated into the floor, vibrational energy harvesting based on the piezoelectric effect may be used instead [16]. By simultaneously extracting energy from diverse sources, a higher amount of harvested energy can be obtained and the time during which no energy is scavenged, can be reduced.

\section{A) SIW antenna as integration platform}

SIW based antenna topologies leverage a ground plane in combination with vias to achieve a high isolation between the radiating element and its environment. This makes them not only very suitable for comfortable integration into garments or unobtrusive embedding into floors or walls, but also facilitates the integration of additional electronic circuitry without degrading their performance. Subsection B) will expand on another reason to select SIW based antennas, being the ease by which additional hardware can be integrated and the ability to minimize the amount and length of interconnections, by exploiting the properties of the topology.

In [10], the potential of multiple antenna topologies as integration platform is examined 
and SIW cavity-backed slot antennas are identified as ideal candidates. Two different SIW cavity-backed slot antennas are therefore presented here. Nevertheless, depending on the application, other SIW based antennas such as the ones discussed in [15], [17] or [18] may also serve as an integration platform. To illustrate this, we propose a novel compact dual-polarized textile SIW antenna topology that is also an ideal integration platform for energy harvesting hardware.

\section{1) SFIT SIW cavity-backed slot antenna}

The first topology under study is the wearable SIW cavity-backed textile slot antenna presented in [13], which is constructed entirely of textile materials for application in SFIT systems. This topology is depicted in Fig. 2a and consists of a conducting feed layer and slot layer, which are patterned in a copper-plated taffeta electrotextile (sheet resistance $0.2 \Omega / \square$ ). The conducting layers are deposited on a dielectric substrate, being a closed cell expanded rubber protective foam layer (dielectric constant $\epsilon_{r}=1.495$ and loss tangent $\tan \delta=0.016$ ) with a thickness of $3.94 \mathrm{~mm}$, typically applied in fire fighter jackets. Both conductive fabric layers are glued to the antenna substrate with thermally-activated adhesive sheets. They are electrically connected to form an SIW cavity by means of brass eyelets (outer diameter $d=4 \mathrm{~mm}$ ), which are spaced closely enough to minimize radiation loss $\left(d_{x} / d \leq 2\right.$ and $\left.d_{y} / d \leq 2\right)$ [11]. The materials from which the on-body antenna is assembled are selected with the greatest care to achieve a flexible, low-profile and conformal design. The SIW cavity is square and a dog-bone shaped slot is cut out in the top conducting layer. The dimensions of the cavity were selected to excite the TE120 cavity mode at $2.45 \mathrm{GHz}$, whereas the dimensions of the slot and its position were chosen to obtain optimal coupling with the excited mode. The length of the grounded coplanar waveguide, used as a feed line, was optimized to improve input matching [13] within the $2.45 \mathrm{GHz}$ ISM-band $(2.40 \mathrm{GHz}-2.4835 \mathrm{GHz})$. More details can be found in [13].

\section{2) SFIT compact dual-polarized SIW antenna}

We propose a novel wearable textile SIW antenna topology that also acts as an ideal integration platform for energy harvesting hardware. Depending on the application, this design might be a more appropriate SFIT antenna. The topology, schematically depicted in Fig. 2b, consists of two rectangular quarter-mode (QM) SIW antennas [18], designated as antennas $\mathrm{A}$ and $\mathrm{B}$, which are mirror images of each other about the YZ-plane. The QMSIW cavities are separated by a common via wall, reducing the total number of vias. The dimensions of both QMSIW cavities are chosen such that the resonance frequency of the TE110 mode is $2.45 \mathrm{GHz}$. Moreover, both QMSIW antennas are designed to be quasi-linearly polarized, along orthogonal directions in broadside. The plane defined by $\phi=\pi / 4 \vee \phi=5 \pi / 4$ is the H-plane for antenna $\mathrm{A}$ and the E-plane for antenna B, whereas the plane defined by $\phi=3 \pi / 4 \vee \phi=7 \pi / 4$ is the E-plane for antenna A and the H-plane for antenna B. Both QMSIW cavities are fed by a coaxial probe that efficiently magnetically couples with the TE110 mode. The probe is positioned along the diagonal of the cavity at the optimal location to achieve a $-10 \mathrm{~dB}$ impedance bandwidth covering the $2.45 \mathrm{GHz}$ ISM-band. Together, both antennas form a compact dual-polarized compound antenna, with ports $\mathrm{A}$ and $\mathrm{B}$. This allows the transceiver of the communication system to exploit polarization diversity to combat fading in multipath propagation 


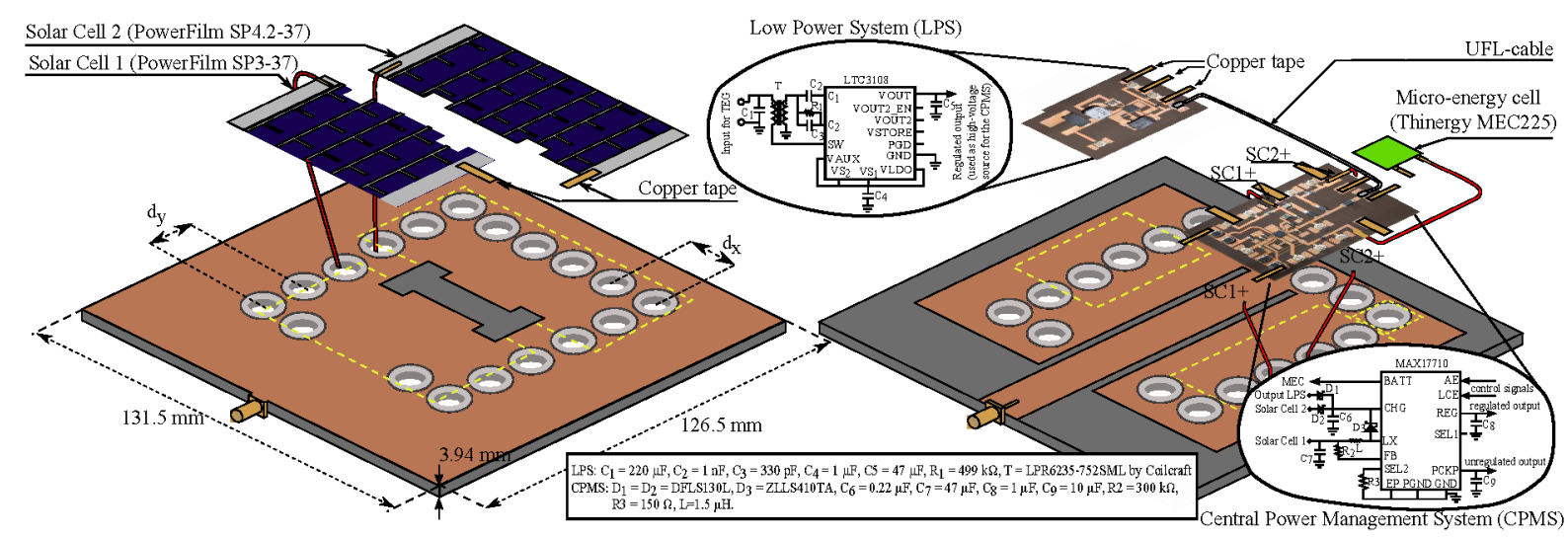

(a)
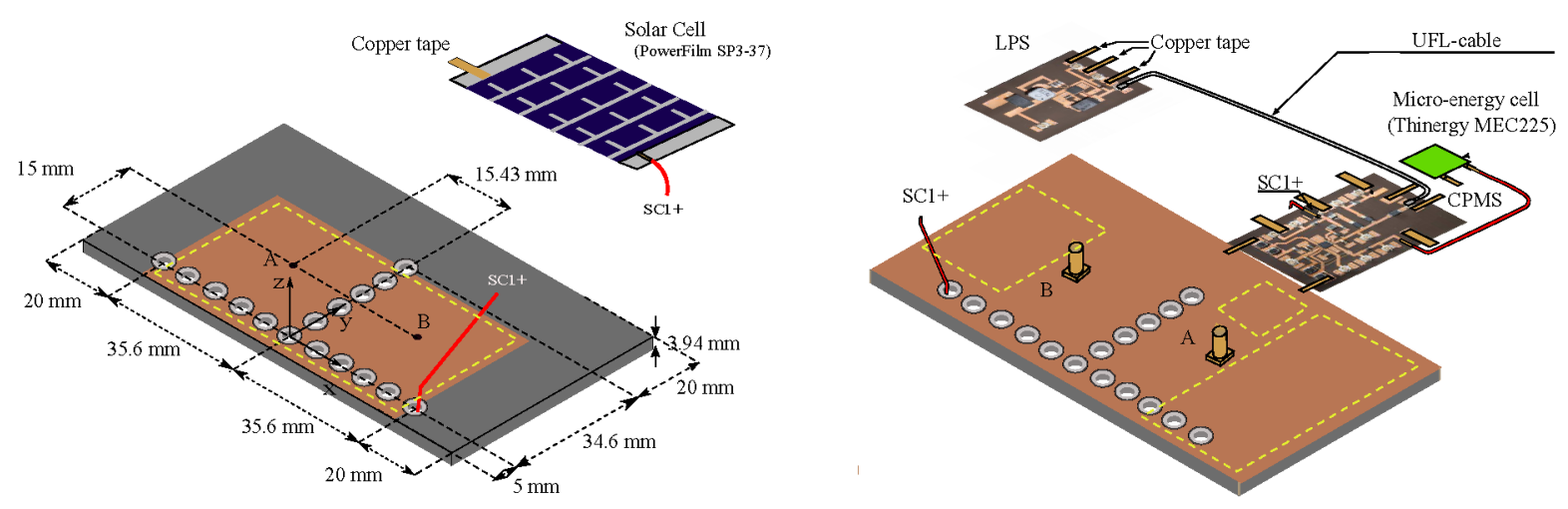

(b)
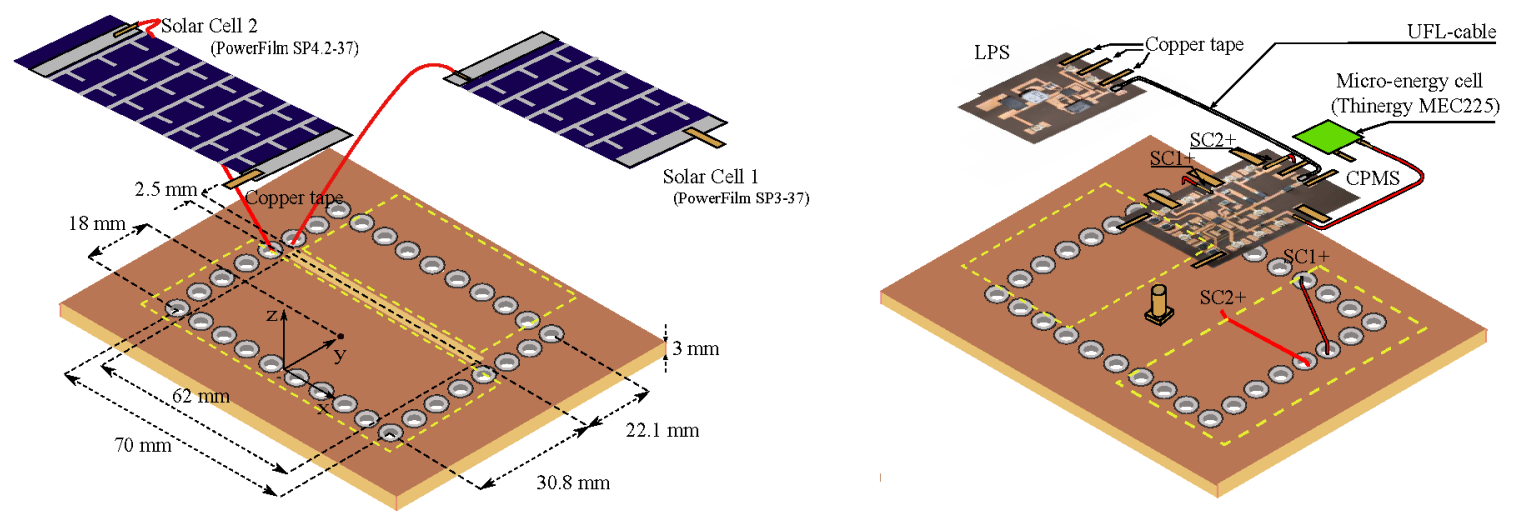

(c)

Figure 2: Integration strategy illustrated on different SIW antenna topologies: one or two flexible a-Si:H solar cells attached to the upper plane (left), and the proposed flexible power management module, including a micro-energy cell, integrated onto their feed plane (right). (a) The wearable SIW cavity-backed textile slot antenna, as described in [13], (b) the novel wearable compact dualpolarized textile SIW antenna, and (c) the SIW cavity-backed slot antenna on cork substrate, as described in [19]. 
environments [20] [21]. The same flexible textile materials as for the SFIT SIW cavity-backed slot antenna are used for the construction, being a closed cell expanded rubber protective foam layer for the antenna substrate and a copper-plated taffeta electrotextile for the conducting layers. Again, brass eyelets are applied, and the stack is assembled with thermally-activated adhesive sheets.

\section{3) IoT UWB SIW cavity-backed slot antenna on cork substrate}

The final topology we discuss is the wideband SIW cavity-backed slot antenna, presented in [19]. Cork is applied as a substrate material, enabling seamless integration in floors or walls. Fig. 2c schematically depicts this topology, which consists of a conducting slot layer and a ground plane, again patterned in copper-plated taffeta electrotextile and glued to the cork substrate with thermally activated adhesive sheets. Appropriately spaced brass eyelets electrically connect the two conducting layers to form the rectangular SIW cavity. The substrate is an ordinary off-the-shelf cork layer for floor or wall finishing with a thickness of $3 \mathrm{~mm}$, provided by Amorim Cork Composites S.A. Its dielectric constant and loss tangent were characterized [19] by a combination of a non-resonant and a resonant method, indicating that the material is slightly inhomogeneous, with an average dielectric constant of $\epsilon_{r}=1.23$ and loss tangent of $\tan \delta=0.0263$. The design is performed using the average parameters, while maintaining some impedance bandwidth margins to allow for varying cork material properties. This topology applies the multi-moding bandwidth enhancement method [22] by using as the radiating element a non-resonant slot in the top patch, much longer than half a wavelength. This almost splits the SIW cavity into two half-cavities, leading to the simultaneous excitation of two hybrid cavity modes. Appropriate dimensioning of the half-cavities and slot leads to resonance frequencies of the two excited hybrid modes that are close together in the frequency band of interest, enlarging the impedance bandwidth. The slotted SIW cavity is fed by a coaxial probe, positioned to achieve a $-10 \mathrm{~dB}$ impedance bandwidth that covers the lower $500 \mathrm{MHz}(3.1 \mathrm{GHz}-3.6 \mathrm{GHz})$ of the $3.1 \mathrm{GHz}-10.6 \mathrm{GHz}$ band, allocated to ultra-wideband (UWB) transmission systems by the Federal Communications Commission [23]. Simulations predict and measurements confirm [19] that the maximum gain and the other far-field properties only vary slightly over the considered frequency band, which is a key requirement to UWB antennas.

\section{B) Energy harvesting and power management hardware}

The discussed SIW antennas are then exploited as hybrid energy harvesting and power management platforms, by deploying a flexible power management module and a $170 \mu \mathrm{m}$-thick MEC (capacity $130 \mu \mathrm{A} \mathrm{h}$ ) on their feed plane and one or two ultra-flexible 200 um-thick PowerFilm thin film a-Si:H solar cells on their upper plane, attached by stretchable adhesive sheets. This is shown schematically in Figs. 2a-2c. The SIW structure of the antennas allows us to minimize the amount and length of interconnections. Therefore, we exploit the SIW cavity as a common DC ground for the energy harvesting hardware and use the hollow eyelets to route the positive connection of the solar cells to the power management system.

The proposed power management system consists of two interconnected subsystems, being the low power system (LPS) and the CPMS. To maintain mechanical flexibility of 
the entire module, the LPS and CPMS are etched on a flexible, ultra-thin polyimide substrate. Moreover, small electronic components were used and distributed over a larger area than necessary. The CPMS is based on the MAX17710 integrated circuit (IC) from Maxim Integrated. It is designed to charge the MEC from two high-voltage DC sources, through linear harvesting, and one low-voltage DC source, via a boost converter. In the meantime, the CPMS protects the MEC and provides a regulated output voltage. Energy harvesting from artificial and solar light is then enabled by connecting solar cell 1 (PowerFilm SP3-37) to the boost converter input and solar cell 2 (PowerFilm SP4.2-37) to a linear harvesting input, respectively. We note that the upper plane of the compact dual-polarized SIW antenna, as shown in Fig. 2b, only provides room for solar cell 1 . The LPS is built around the LTC3108 IC by Linear Technology. It implements an ultra-low voltage step up converter, with its output connected to the second linear harvesting input of the CPMS, to enable thermal body energy harvesting via an externally connected TEG. The TEG is not integrated onto the antenna, to obtain a higher degree of freedom. In this work, a single TEG is connected to the LPS. Specifically, the UltraTec Series UT6,24,F1,5555 component by Laird Technologies is selected [10]. Its hot side is tightly attached to the skin of the wearer's arm, whereas its cold side is exposed to the ambient air, in order to harvest thermal body energy based on Seebeck's effect [8]. However, multiple TEGs, potentially stacked, or a heat sink could be used to further increase the amount of harvested energy. In a floor or wall integration scenario, being the typical deployment configuration of the UWB SIW cavity-backed slot antenna on cork substrate, thermal energy harvesting may not be relevant. Then, vibrational energy harvesting based on the piezoelectric effect may be used instead [16].

To ensure stable performance of the SIW antennas after hardware integration, the solar cells are attached to the conducting parts of the antenna without extending beyond them, and without covering the radiating element. In the case of the textile SIW cavity-backed slot antenna (Fig. 2a), the two flexible solar cells were therefore patterned to fit the antenna's slot. Furthermore both flexible circuit boards were oriented such that the feed line remains uncovered. Both the compact dual-polarized SIW antenna (Fig. 2b) and the UWB SIW cavity-backed slot antenna on cork substrate (Fig. 2c) are fed by a coaxial probe, and they use either a rectangular slot, or no slot at all, making the above considerations irrelevant for them.

\section{MEASUREMENT RESULTS}

\section{A) Figures of merit of the SIW antennas}

First, the integration procedure is validated by verifying that the performance of the SIW antennas, discussed in Section II, remains nearly undisturbed by the integrated hardware. Therefore, the reflection coefficient and the far-field gain pattern of all discussed SIW antennas were measured and compared at different stages of hardware integration, more specifically before the integration of any hardware, after solar cell integration, and when all energy harvesting and power management hardware is integrated and all connections are made according to Figs. 2a-2c. All measurements are conducted inside an anechoic room mimicking free-space conditions, by means of an Agilent N5242A PNA-X Microwave Network Analyzer and an Orbit/FR DBDR antenna positioning system. The 


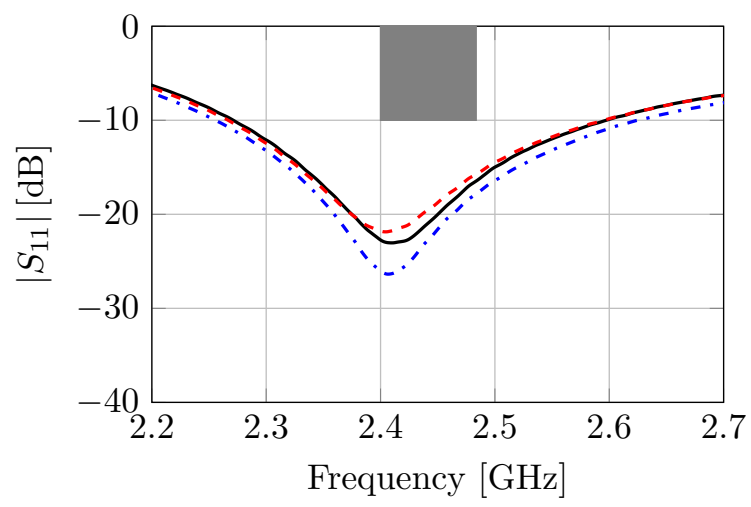

Figure 3: Measured input reflection coefficient $\left|S_{11}\right|[\mathrm{dB}]$ versus frequency of the wearable SIW cavity-backed slot antenna for different stages of hardware integration. Undisturbed antenna (-), solar cells integrated $(---)$, and all energy harvesting hardware integrated $(-\cdot-\cdot)$.

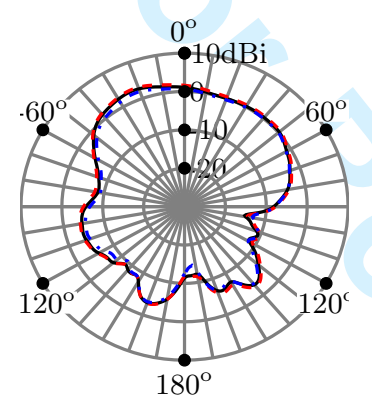

(a)

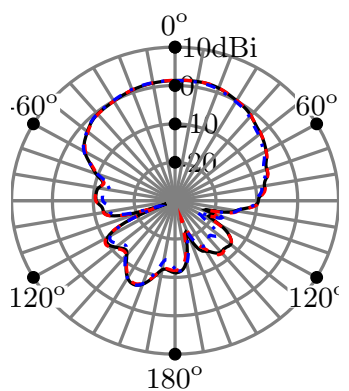

(b)

Figure 4: Measured far-field gain pattern $[\mathrm{dBi}]$ of the wearable SIW cavity-backed slot antenna at $2.45 \mathrm{GHz}$, in both (a) the E-plane and (b) the H-plane. Undisturbed antenna (-), solar cells integrated $(---)$, and all energy harvesting hardware integrated $(-\cdot-\cdot)$.

far-field gain patterns are measured by the gain comparison method using the MI-12 standard gain horn series.

\section{1) SFIT SIW cavity-backed slot antenna}

Fig. 3 shows the input reflection coefficient from $2.2 \mathrm{GHz}$ to $2.7 \mathrm{GHz}$, whereas Fig. 4 shows the gain patterns in the E- and $\mathrm{H}$-plane at $2.45 \mathrm{GHz}$, for all three stages of hardware integration. These measurements show that the integration of the solar cells, the flexible power management system and MEC, only has a negligible influence on the antenna's performance. Hence, they prove that this topology is indeed very suitable as an integration platform, reducing the amount and length of interconnecting wires by exploiting its excellent antenna/environment isolation. The antenna's outstanding on-body performance was already demonstrated in [13]. 


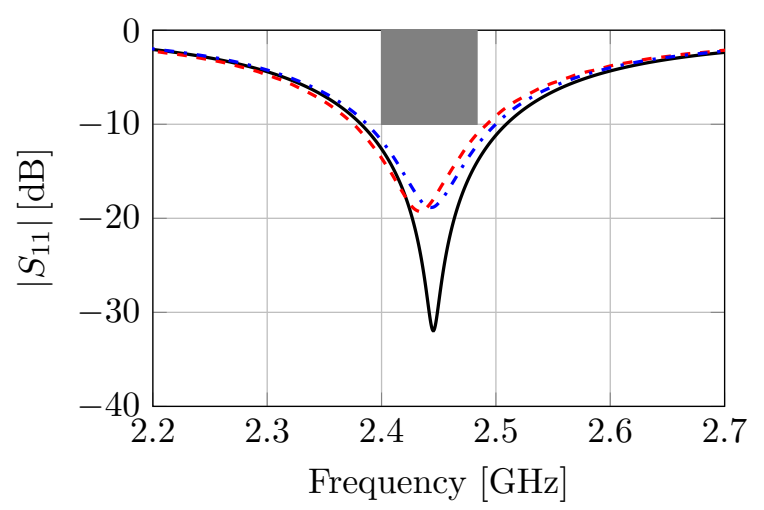

Figure 5: Simulated (-) and measured input reflection coefficient $\left|S_{11}\right|[\mathrm{dB}]$ versus frequency for ports A $(---)$ and B $(-\cdot-\cdot)$ of the dual-polarized SIW antenna.

\section{2) SFIT compact dual-polarized SIW antenna}

Fig. 5 compares the simulated and measured free-space input reflection coefficients, for both ports A and B. The slight difference is caused by fabrication inaccuracies. The impedance bandwidth of both antenna ports covers the entire $2.45 \mathrm{GHz}$ ISM-band $(2.40 \mathrm{GHz}-2.4835 \mathrm{GHz})$. Fig. 6 shows that the isolation between port A and B is larger than $14 \mathrm{~dB}$ in the entire $2.45 \mathrm{GHz}$ ISM-band. Figs. 7 a to $7 \mathrm{~d}$ show the simulated and measured far-field gain patterns for ports A and B, in the E-plane and H-plane at $2.45 \mathrm{GHz}$. The most important performance parameters, measured at $2.45 \mathrm{GHz}$ for both ports $\mathrm{A}$ and $\mathrm{B}$, are listed and compared to the simulation values in Table 1 . These include the radiation efficiency, the FTBR (defined as the ratio of the gain in the $+z$ direction to the gain in the $-z$ direction), the maximum gain, and the polarization properties of the broadside radiation, being the tilt angle $\alpha$ of the polarization ellipse (defined as the angle between the x-axis and the major axis of the ellipse), and its axial ratio (defined as the ratio of the major axis to the minor axis with a positive sign indicating right-hand elliptical polarization). In general, a good agreement between measurement and simulation is observed. We again confirm that the integrated hardware does not disturb the properties of the SIW antenna. Indeed, the input reflection coefficient for port A, shown in Fig. 8, and the far-field gain patterns in the E- and $\mathrm{H}$-plane at $2.45 \mathrm{GHz}$ for port A, shown in Fig. 9, are nearly identical for all three stages of hardware integration. Table 1 also lists the radiation efficiency, FTBR, maximum gain and broadside polarization properties for port A, at different stages of hardware integration. Only the axial ratio is slightly degraded by the integrated hardware, while the other values remain constant, within the experimental error.

\section{3) IoT UWB SIW cavity-backed slot antenna on cork substrate}

Fig. 10 shows the input reflection coefficient from $2.8 \mathrm{GHz}$ to $4.0 \mathrm{GHz}$, whereas Fig. 11 shows the gain patterns in the E- and H-plane at the lower frequency $3.10 \mathrm{GHz}$, the center frequency $3.35 \mathrm{GHz}$ and the upper frequency $3.60 \mathrm{GHz}$, for all three stages of hardware integration. These measurements confirm that the hardware integration only has a minor influence on the antenna's performance and that this topology serves as an excellent 


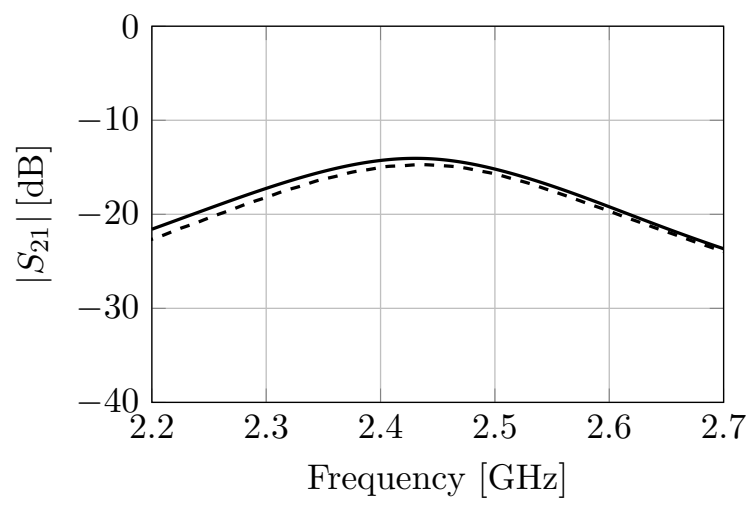

Figure 6: Simulated (-) and measured (-- ) transmission coefficient $\left|S_{21}\right|[\mathrm{dB}]$ versus frequency between ports A and B of the dual-polarized SIW antenna.

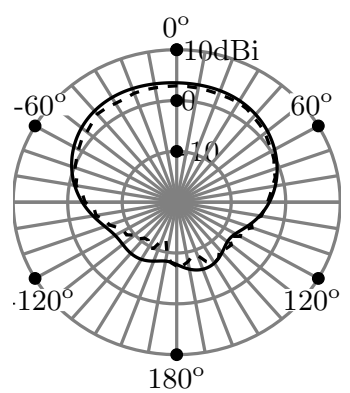

(a)

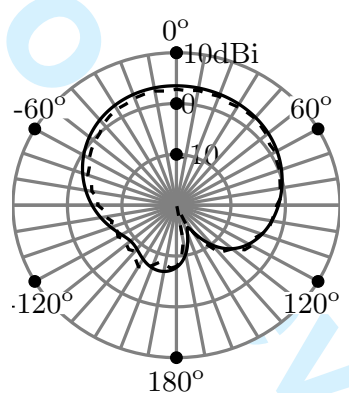

(b)

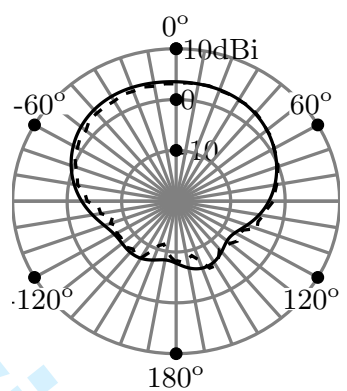

(c)

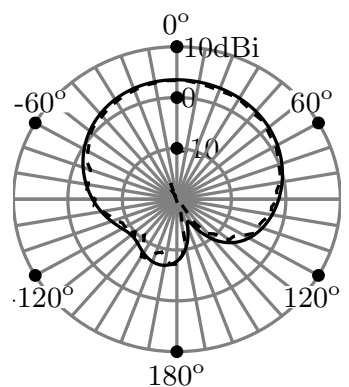

(d)

Figure 7: Far-field gain pattern [dBi] of the dual-polarized SIW antenna at $2.45 \mathrm{GHz}$, for port A in (a) the E-plane and (b) the H-plane, and for port B in (c) the E-plane and (d) the H-plane. Simulation $(-)$ and measurement $(---)$.

Table 1: Measured (simulated) antenna characteristics at $2.45 \mathrm{GHz}$ for ports $\mathrm{A}$ and $\mathrm{B}$ of the dual-polarized SIW antenna.

\begin{tabular}{|c||c||c||c||c|}
\hline Parameter & Port A & Port B & $\begin{array}{c}\text { Port A } \\
\text { with solar cell }\end{array}$ & $\begin{array}{c}\text { Port A } \\
\text { with all hardware }\end{array}$ \\
\hline \hline Radiation efficiency [\%] & $71(73)$ & $78(73)$ & $71(-)$ & $72(-)$ \\
\hline FTBR [dB] & $11.0(11.2)$ & $10.8(11.3)$ & $10.5(-)$ & $10.6(-)$ \\
\hline Maximum gain [dBi] & $3.6(4.1)$ & $3.9(4.1)$ & $3.5(-)$ & $3.5(-)$ \\
\hline Tilt angle $\alpha$ [deg.] & $138.4(134.7)$ & $46.0(45.3)$ & $137.4(-)$ & $139.2(-)$ \\
\hline Axial ratio [-] & $-9.9(-15.7)$ & $13.4(15.6)$ & $-6.7(-)$ & $-7.8(-)$ \\
\hline
\end{tabular}

hardware integration platform. 


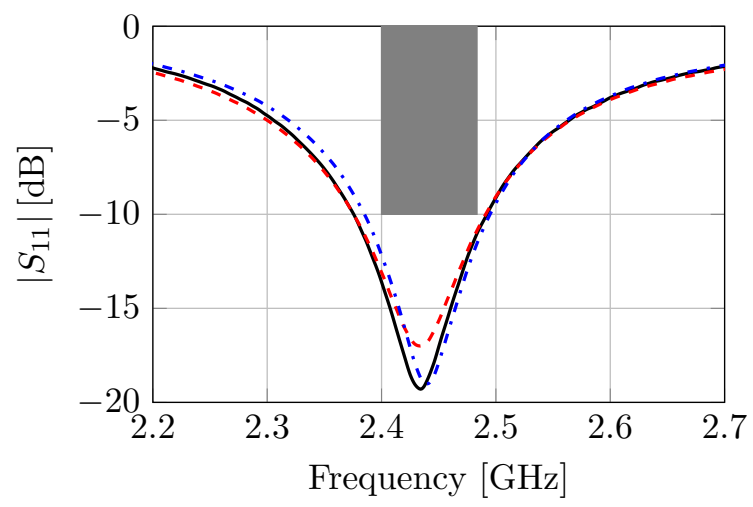

Figure 8: Measured input reflection coefficient $\left|S_{11}\right|[\mathrm{dB}]$ versus frequency for port A of the dualpolarized SIW antenna for different stages of hardware integration. Undisturbed antenna (-), solar cell integrated $(---)$, and all energy harvesting hardware integrated $(-\cdot-\cdot)$.

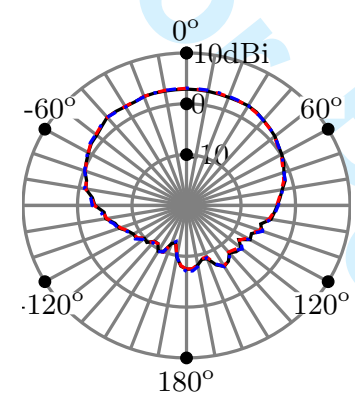

(a)

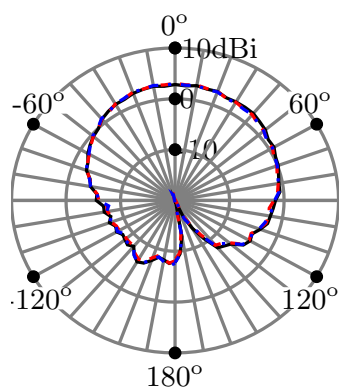

(b)

Figure 9: Measured far-field gain pattern [dBi] for port A of the dual-polarized SIW antenna at $2.45 \mathrm{GHz}$, in both (a) the E-plane and (b) the H-plane. Undisturbed antenna ( - ), solar cell integrated $(---)$, and all energy harvesting hardware integrated $(-\cdot-\cdot)$.

\section{B) Energy supplied by the hybrid energy-harvesting system}

Finally, four well-chosen scenarios, each lasting 20 minutes, are studied in a realistic indoor environment to demonstrate the energy harvesting potential of our system in low power density situations. In each scenario, a test person, with a height of $1.87 \mathrm{~m}$, wears the compact, highly-integrated and unobtrusive unit on his chest and the selected TEG on his left arm. During each 20-minutes interval, the power scavenged by each of the three energy harvesters is measured as well as the effectively delivered power to the MEC. Yet, only the power delivered by solar cell 1, the TEG and the sum of both are depicted in Figs. 12a to 12d, as it turned out that solar cell 2, in combination with the linear harvesting technique, was not able to harvest energy in these indoor scenarios.

The first scenario comprises a test person sitting in a $2.4 \mathrm{~m}$ high room with a uniform temperature of $15^{\circ} \mathrm{C}$, no active light sources and no air circulation. Fig. 12a shows that the thermal gradient between room and skin temperature is high enough to harvest energy via the TEG, whereas the absence of light prevents solar cell 1 from scavenging energy. The peaks in scavenged energy by the TEG can be attributed to stretching of the 


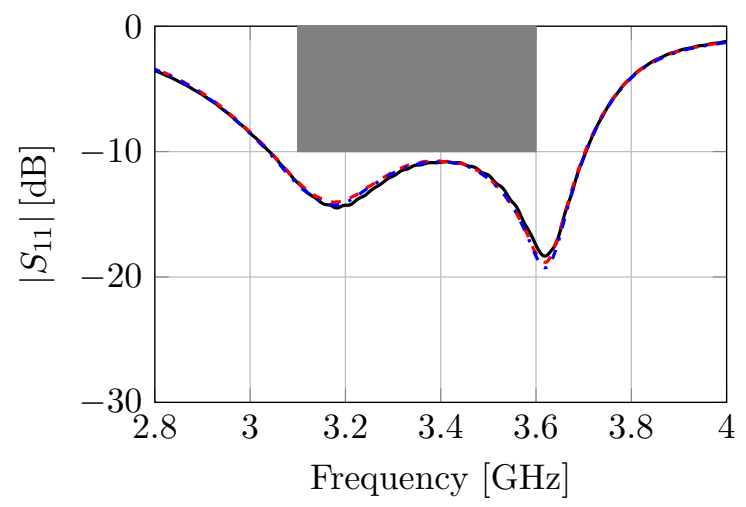

Figure 10: Measured input reflection coefficient $\left|S_{11}\right|[\mathrm{dB}]$ versus frequency of the SIW cavitybacked slot antenna on cork substrate for different stages of hardware integration. Undisturbed antenna $(-)$, solar cells integrated $(---)$, and all energy harvesting hardware integrated $(-\cdot-\cdot)$.

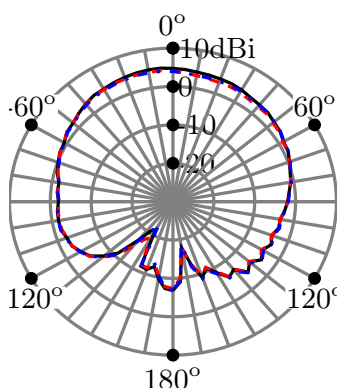

(a)

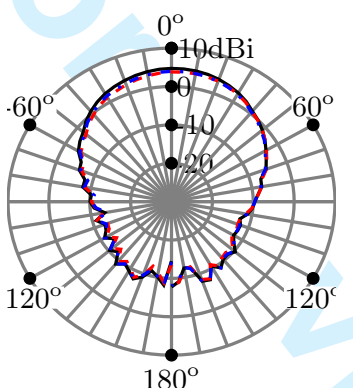

(b)

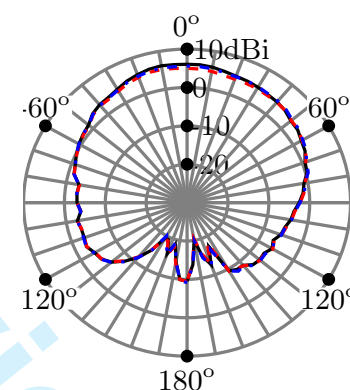

(c)

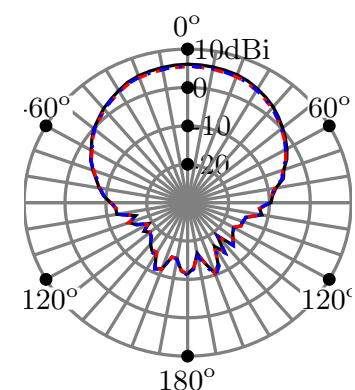

(d)

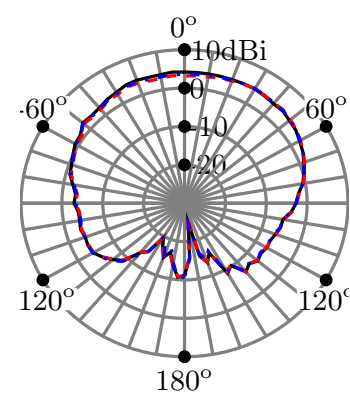

(e)

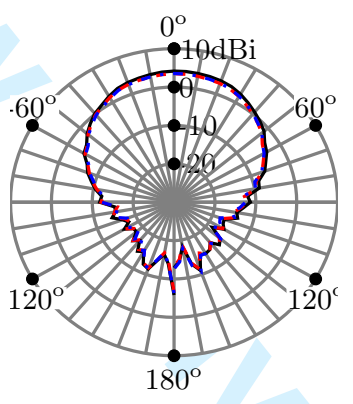

(f)

Figure 11: Measured far-field gain pattern [dBi] of the SIW cavity-backed slot antenna on cork substrate at the lower frequency $3.10 \mathrm{GHz}$, in (a) the E-plane and (b) the H-plane, at the center frequency $3.35 \mathrm{GHz}$, in (c) the E-plane and (d) the H-plane, and at the upper frequency $3.60 \mathrm{GHz}$, in (e) the E-plane and (f) the H-plane. Undisturbed antenna (-), solar cells integrated (-- $)$, and all energy harvesting hardware integrated $(-\cdot-\cdot)$.

wearer. On average, the TEG was able to generate $108.6 \mu \mathrm{W}$, of which $39.2 \mu \mathrm{W}$ was effectively delivered to the MEC, leading to an overall system efficiency $\eta$ of $36.1 \%$.

The second scenario is very similar to the first scenario. However, in the second scenario, 

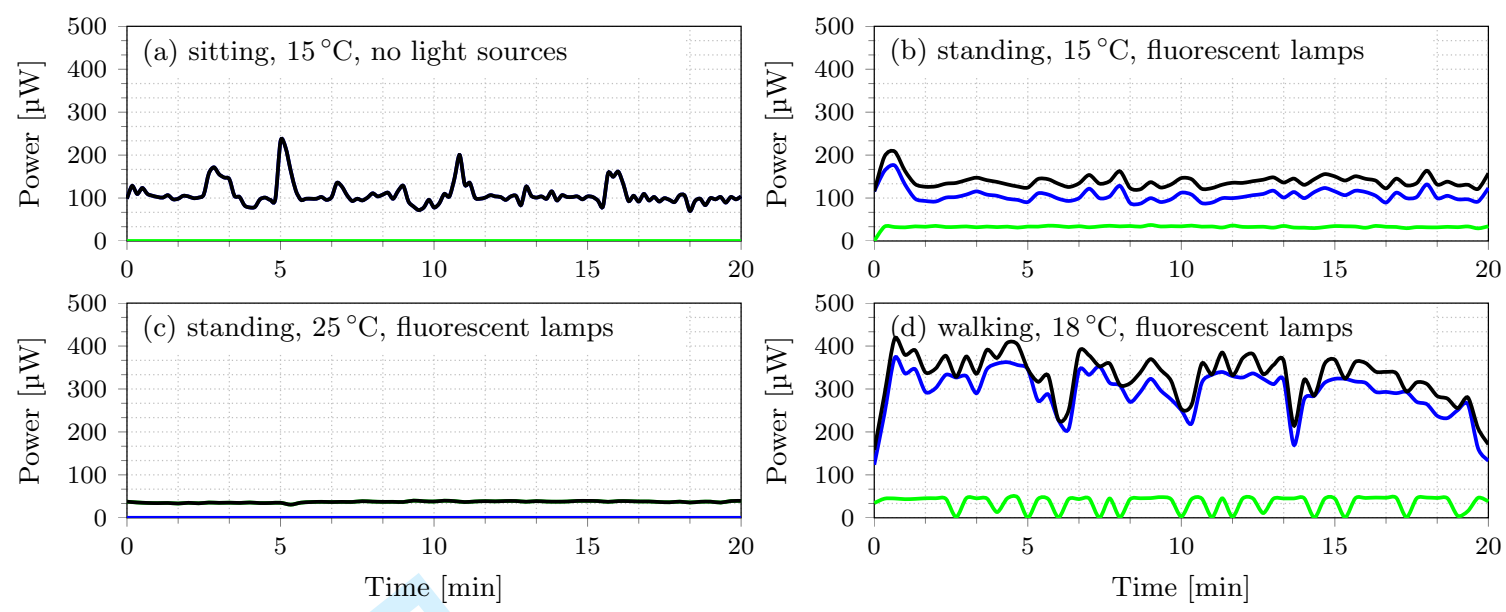

Figure 12: Power generated by the TEG (blue solid line), solar cell 1 (green solid line) and the sum of both (black solid line), as a function of time, for each of the four considered realistic indoor scenarios.

fluorescent lamps mounted at the ceiling are turned on and the test person is standing in an upright position. As shown in Fig. 12b, the TEG and solar cell 1 are now both able to harvest energy. On average, $106.7 \mu \mathrm{W}$ is recovered by the TEG and $33.2 \mu \mathrm{W}$ by solar cell 1 , resulting in a total harvested power of $139.9 \mu \mathrm{W}$. Eventually, $60.6 \mu \mathrm{W}$ was stored in the MEC, corresponding to $\eta=43.2 \%$.

In the third scenario, the room is heated up to $25^{\circ} \mathrm{C}$. Fig. $12 \mathrm{c}$ indicates that the room temperature is too high for the TEG to recover energy and that only solar cell 1 is capable of harvesting energy. Solar cell 1 produced $37.0 \mu \mathrm{W}$ on average, of which $24.4 \mu \mathrm{W}$ is effectively stored by the MEC.

In scenario 4 , the test person is walking in a corridor with a height of $2.2 \mathrm{~m}$, a steady temperature of $18{ }^{\circ} \mathrm{C}$, no air circulation and similar fluorescent lights at the ceiling.

Fig. 12d clearly demonstrates that, as a result of the dynamic nature of the wearer and the corresponding increase in convection, the power generation by the TEG is substantially increased. Drops in generated power by the TEG and solar cell 1 can be attributed to pauses in which the test person stops walking and the distance between subsequent lamps, respectively. During this 20 -minutes interval, $296.0 \mu \mathrm{W}$ and $34.8 \mu \mathrm{W}$ was generated by the TEG and solar cell 1 on average, respectively. This resulted in a total average power of $152.17 \mu \mathrm{W}$ that could be effectively harvested, leading to $\eta=46.0 \%$. Figs. 12a to 12d clearly evince the necessity of hybrid energy harvesting in real-life scenarios. Figs. 12a and 12c show that no power would have been harvested if the user only relied on either solar cell 1 or the TEG, respectively. Hence, it reduces the periods during which no energy is harvested. Fig. 12b depicts that hybrid energy harvesting may result in a higher amount of scavenged energy by combining the energy harvested from diverse energy sources. Both effects could lead to either a higher system autonomy or a reduced battery size. An even higher amount of energy could be recovered by solar cell 1 and the TEG in outdoor environments thanks to improved convection and light conditions. In addition, in such an environment, solar cell 2 is also able to scavenge energy. More specifically, in case of a turbidity-free sky, placing solar cell 2 perpendicular 
to the earth surface, pointed in the direction of the sun should enable harvesting an additional $49 \mathrm{~mW}[10]$.

\section{CONCLUSION}

In this paper, we discussed the integration on an SIW antenna of up to three energy harvesters, including two flexible solar cells and a thermoelectric generator, together with a micro-energy cell and a corresponding flexible power management system. The total system is intended for garment integration or embedding in floor or wall materials. Two different SIW cavity-backed slot antennas and a novel compact dual linearly polarized SIW antenna topology were proposed to serve as platforms for this additional hardware. The pursued integration procedure minimizes the hardware's influence on antenna performance and, in the meantime, guarantees wearability or unobtrusive floor/wall integration. Measurements prove that the selected SIW antennas are indeed suitable as integration platforms, whereas experiments in four carefully-selected realistic indoor environments demonstrated that a hybrid energy-harvesting approach leads to a higher amount of energy, scavenged in a more continuous way.

\section{ACKNOWLEDGEMENTS}

Part of the work was supported by the iMinds IoT research program.

\section{REFERENCES}

[1] F. Mattern and C. Floerkemeier, "From Active Data Management to Event-based Systems and More," K. Sachs, I. Petrov, and P. Guerrero, Eds. Berlin, Heidelberg: Springer-Verlag, 2010, ch. From the Internet of Computers to the Internet of Things, pp. 242-259.

[2] A. Dierck, S. Agneessens, F. Declercq, B. Spinnewyn, G.-J. Stockman, P. Van Torre, L. Vallozzi, D. Vande Ginste, J. Vanfleteren, T. Vervust, and H. Rogier, "Active textile antennas in professional garments for sensing, localisation and communication," International Journal of Microwave and Wireless Technologies, vol. 6, no. 3-4, pp. 331-341, 2014.

[3] M. A. Hanson, H. C. Powell Jr, A. T. Barth, K. Ringgenberg, B. H. Calhoun, J. H. Aylor, and J. Lach, "Body Area Sensor Networks: Challenges and Opportunities," Computer, vol. 42, no. 1, pp. 58-65, 2009.

[4] L. Huang, V. Pop, R. de Francisco, R. Vullers, G. Dolmans, H. de Groot, and K. Imamura, "Ultra Low Power Wireless and Energy Harvesting Technologies - An Ideal Combination," in Proc. IEEE Int. Conf. Commun. Syst., Singapore, 17-19 Nov. 2010, pp. 295-300.

[5] P. Van Torre, L. Vallozzi, A. Dierck, H. Rogier, and M. Moeneclaey, "Power-efficient Bodycentric Communications," in URSI Benelux Forum, 14 Sept. 2012, pp. 8-10. 
[6] A. Collado and A. Georgiadis, "Conformal Hybrid Solar and Electromagnetic (EM) Energy Harvesting Rectenna," IEEE Trans. Circuits Syst. I, Reg. Papers, vol. 60, no. 8, pp. 22252234, Aug 2013.

[7] S. Lemey, F. Declercq, and H. Rogier, "Dual-Band Substrate Integrated Waveguide Textile Antenna With Integrated Solar Harvester," IEEE Antennas Wireless Propag. Lett., vol. 13, no. 1, pp. 269-272, 2014.

[8] M. Lossec, B. Multon, H. Ahmed, and C. Goupil, "Thermoelectric Generator Placed on the Human Body: System Modeling and Energy Conversion Improvements," Eur. Phys. J, vol. 52 , no. 1 , pp. 1-10, 2010.

[9] M. Virili, A. Georgiadis, K. Niotaki, A. Collado, F. Alimenti, P. Mezzanotte, L. Roselli, and N. B. Carvalho, "Design and optimization of an antenna with Thermo-Electric Generator (TEG) for autonomous wireless nodes," 2014 IEEE RFID Technology and Applications Conference (RFID-TA), pp. 21-25, Sep 8-9 Sept. 2014.

[10] S. Lemey, F. Declercq, and H. Rogier, "Textile Antennas as Hybrid Energy-Harvesting Platforms," Proceedings of the IEEE, vol. 102, no. 11, pp. 1833-1857, Nov 2014.

[11] M. Bozzi, A. Georgiadis, and K. Wu, "Review of substrate-integrated waveguide circuits and antennas," IET Microwaves, Antennas \& Propagation, vol. 5, no. 8, pp. 909-920, June 2011.

[12] G. Q. Luo, T. Y. Wang, and X. H. Zhang, "Review of Low Profile Substrate Integrated Waveguide Cavity Backed Antennas," International Journal of Antennas and Propagation, vol. 2013, 7 pages. [Online]. Available: http://dx.doi.org/10.1155/2013/746920

[13] R. Moro, S. Agneessens, H. Rogier, and M. Bozzi, "Wearable Textile Antenna in Substrate Integrated Waveguide Technology," IET Electron. Lett., vol. 48, no. 16, pp. 985-987, 2012.

[14] R. Moro, S. Agneessens, H. Rogier, A. Dierck, and M. Bozzi, "Textile Microwave Components in Substrate Integrated Waveguide Technology," IEEE Transactions on Microwave Theory and Techniques, vol. 63, no. 2, pp. 422-432, February 2015.

[15] O. Caytan, S. Lemey, S. Agneessens, D. Vande Ginste, P. Demeester, C. Loss, R. Salvado, and H. Rogier, "Half-Mode Substrate-Integrated-Waveguide Cavity-Backed Slot Antenna on Cork Substrate," IEEE Antennas and Wireless Propagation Letters, in press 2015. [Online]. Available: http://dx.doi.org/10.1109/LAWP.2015.2435891

[16] Y. Jeon, R. Sood, J. h. Jeong, and S.-G. Kim, "MEMS power generator with transverse mode thin film PZT," Sensors and Actuators A: Physical, vol. 122, no. 1, pp. 16 - 22, 2005.

[17] S. Agneessens, S. Lemey, R. Moro, M. Bozzi, and H. Rogier, "The next generation textile antennas based on substrate integrated waveguide technology," in Proc. of the XXXIth General Assembly and Scientific Symposium of the International Union of Radio Science, Beijing, China, 2014, pp. 1-4.

[18] C. Jin, R. Li, A. Alphones, and X. Bao, "Quarter-Mode Substrate Integrated Waveguide and Its Application to Antennas Design," IEEE Transactions on Antennas and Propagation, vol. 61, no. 6, pp. 2921-2928, June 2013. 
[19] O. Caytan, S. Agneessens, S. Lemey, D. Vande Ginste, P. Demeester, and H. Rogier, "UltraWideband Cork Substrate-Integrated-Waveguide Cavity-Backed Slot Antenna," in 2015 International Conference on Electromagnetics in Advanced Applications (ICEAA), Sept 2015, pp. 1104-1107.

[20] L. Vallozzi, P. Van Torre, C. Hertleer, H. Rogier, M. Moeneclaey, and J. Verhaevert, "Wireless Communication for Firefighters Using Dual-Polarized Textile Antennas Integrated in Their Garment," IEEE Transactions on Antennas and Propagation, vol. 58, no. 4, pp. 1357-1368, April 2010.

[21] P. Van Torre, L. Vallozzi, C. Hertleer, H. Rogier, M. Moeneclaey, and J. Verhaevert, "Indoor Off-Body Wireless MIMO Communication With Dual Polarized Textile Antennas," IEEE Transactions on Antennas and Propagation, vol. 59, no. 2, pp. 631-642, Feb 2011.

[22] G. Q. Luo, Z. F. Hu, W. J. Li, X. H. Zhang, L. L. Sun, and J. F. Zheng, "BandwidthEnhanced Low-Profile Cavity-Backed Slot Antenna by Using Hybrid SIW Cavity Modes," IEEE Transactions on Antennas and Propagation, vol. 60, no. 4, pp. 1698-1704, April 2012.

[23] D. Dardari, R. D'Errico, C. Roblin, A. Sibille, and M. Win, "Ultrawide Bandwidth RFID: The Next Generation?" Proceedings of the IEEE, vol. 98, no. 9, pp. 1570-1582, September 2010 .

\section{Bibliographies}

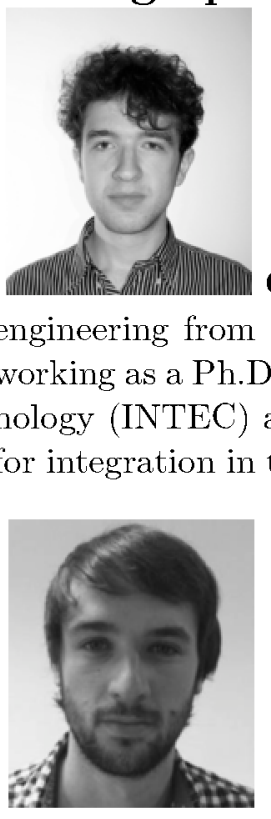

Olivier Caytan was born in 1990. He received the M.Sc. degree in electrical engineering from Ghent University, Ghent, Belgium in 2014. Since October 2014, he has been working as a Ph.D. student at the Electromagnetics group in the Department of Information Technology (INTEC) at Ghent University. His research focuses on the design of low profile antennas for integration in textile, floor or wall materials, in the context of Internet of Things applications.

(an Lemey was born in 1990. He received the M.Sc. degree in electronic engineering from Ghent University, Campus Kortrijk, Belgium, in 2012. Since September 2012, he has been working toward the Ph.D. degree in the Electromagnetics Group, Department of Information Technology (INTEC), Ghent University. His research activities focus on the design of textile antennas for smart fabric interactive textile systems, the reuse of textile antennas as hybrid energy harvesting and power management platforms, new techniques for implementing substrate integrated waveguide (SIW) structures in textile materials, and ultra-short-range multiple-input 
multiple-output wireless communication (MIMO).

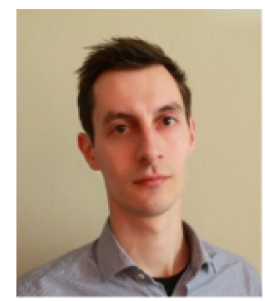

Sam Agneessens (M 2014) was born in Belgium in 1986. He received the M.S. and $\mathrm{PhD}$ degrees in electrical engineering from the University of Ghent, Ghent, Belgium, in 2011 and 2015. He is currently an FWO postdoctoral fellow within the Electromagnetics group in the Department of Information Technology (INTEC) at Ghent University. His research focuses on the design and development of textile antennas for wearable applications and integrated mm-wave antennas. Sam Agneessens received the URSI Young Scientist Award at the 2014 URSI General Assembly and is co-recipient of the 2014 Premium Award for Best Paper in IET Electronics Letters.

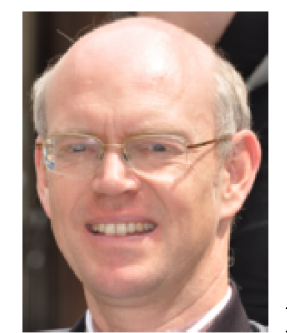

Hendrik Rogier received the Electrical Engineering and the Ph.D. degrees from Ghent University, Gent, Belgium, in 1994 and in 1999, respectively. He is a Full Professor at the Department of Information Technology, iMinds/Ghent University. From October 2003 to April 2004, he was Visiting Scientist at the Mobile Communications Group of Vienna University of Technology. He authored and coauthored about 125 papers in international journals and about 140 contributions in conference proceedings. He is Associate Editor of IET Electronics Letters and a member of the Editorial Board of IET Science, Measurement Technology. His current research interests are antenna systems, radiowave propagation, body-centric communication, numerical electromagnetics, electromagnetic compatibility and power/signal integrity. He was twice awarded the URSI Young Scientist Award. Moreover, he received the 2014 Premium Award for Best Paper in IET Electronics Letters and several awards at conferences. He is a Senior Member of the IEEE.

\section{List of figures and tables}

Fig. 1. Block schematic representation of system concept and application.

Fig. 2. Integration strategy illustrated on different SIW antenna topologies: one or two flexible a-Si:H solar cells attached to the upper plane (left), and the proposed flexible power management module, including a micro-energy cell, integrated onto their feed plane (right). (a) The wearable SIW cavity-backed textile slot antenna, as described in [13], (b) the novel wearable compact dualpolarized textile SIW antenna, and (c) the SIW cavity-backed slot antenna on cork substrate, as described in [19].

Fig. 3. Measured input reflection coefficient $\left|S_{11}\right|[\mathrm{dB}]$ versus frequency of the wearable SIW cavity-backed slot antenna for different stages of hardware integration. Undisturbed antenna, solar cells integrated, and all energy harvesting hardware integrated. 
Fig. 4. Measured far-field gain pattern $[\mathrm{dBi}]$ of the wearable SIW cavity-backed slot antenna at $2.45 \mathrm{GHz}$, in both (a) the E-plane and (b) the H-plane. Undisturbed antenna, solar cells integrated, and all energy harvesting hardware integrated.

Fig. 5. Simulated and measured input reflection coefficient $\left|S_{11}\right|[\mathrm{dB}]$ versus frequency for ports $\mathrm{A}$ and $\mathrm{B}$ of the dual-polarized SIW antenna.

Fig. 6. Simulated and measured transmission coefficient $\left|S_{21}\right|[\mathrm{dB}]$ versus frequency between ports A and B of the dual-polarized SIW antenna.

Fig. 7. Far-field gain pattern [dBi] of the dual-polarized SIW antenna at $2.45 \mathrm{GHz}$, for port A in (a) the E-plane and (b) the H-plane, and for port B in (c) the E-plane and (d) the H-plane. Simulation and measurement.

Table 1. Measured (simulated) antenna characteristics at $2.45 \mathrm{GHz}$ for ports A and B of the dual-polarized SIW antenna.

Fig. 8. Measured input reflection coefficient $\left|S_{11}\right|[\mathrm{dB}]$ versus frequency for port A of the dualpolarized SIW antenna for different stages of hardware integration. Undisturbed antenna, solar cell integrated, and all energy harvesting hardware integrated.

Fig. 9. Measured far-field gain pattern [dBi] for port A of the dual-polarized SIW antenna at $2.45 \mathrm{GHz}$, in both (a) the E-plane and (b) the H-plane. Undisturbed antenna, solar cell integrated, and all energy harvesting hardware integrated.

Fig. 10. Measured input reflection coefficient $\left|S_{11}\right|[\mathrm{dB}]$ versus frequency of the SIW cavity-backed slot antenna on cork substrate for different stages of hardware integration. Undisturbed antenna, solar cells integrated, and all energy harvesting hardware integrated.

Fig. 11. Measured far-field gain pattern [dBi] of the SIW cavity-backed slot antenna on cork substrate at the lower frequency $3.10 \mathrm{GHz}$, in (a) the E-plane and (b) the H-plane, at the center frequency $3.35 \mathrm{GHz}$, in (c) the E-plane and (d) the H-plane, and at the upper frequency $3.60 \mathrm{GHz}$, in (e) the E-plane and (f) the H-plane. Undisturbed antenna, solar cells integrated, and all energy harvesting hardware integrated.

Fig. 12. Power generated by the TEG (blue solid line), solar cell 1 (green solid line) and the sum of both (black solid line), as a function of time, for each of the four considered realistic indoor scenarios. 